\title{
Exploring Factors Influencing Participant Drop-Out Behavior in a Living Lab Environment
}

\author{
Abdolrasoul Habibipour ${ }^{(\bowtie)}$, Ali Padyab, Birgitta Bergvall-Kåreborn, \\ and Anna Ståhlbröst \\ Information Systems, Luleå University of Technology, Luleå, Sweden \\ \{Abdolrasoul. Habibipour, Ali. Padyab, \\ Birgitta. Bergvall-Kareborn, Anna.Stahlbrost\}@ltu.se
}

\begin{abstract}
The concept of "living lab" is a rather new phenomenon that facilitates user involvement in open innovation activities. The users' motivations to contribute to the living lab activities at the beginning of the project are usually higher than once the activities are underway. However, the literature still lacks an understanding of what actions are necessary to reduce the likelihood of user drop-out throughout the user engagement process. This study aims to explore key factors that are influential on user drop-out in a living lab setting by engaging users to test an innovation during the pilot phase of the application's development. The stability of the prototype, ease of use, privacy protection, flexibility of the prototype, effects of reminders, and timing issues are the key influential factors on user drop-out behavior. This paper summarizes the key lessons learned from the case study and points to avenues for future research.
\end{abstract}

Keywords: User engagement $\cdot$ Drop-out $\cdot$ Living lab $\cdot$ Case study $\cdot$ Field test

\section{Introduction}

Open innovation by involving individual users within the process of information systems development (ISD) contributes positively to new innovations [1] as well as system success, system acceptance, and user satisfaction [2, 3]. Open innovation assumes that "firms can and should use external ideas as well as internal ideas, and internal and external paths to market, as firms look to advance their technology" [4]. In this regard, a living lab is a way of managing open innovation in which individual users are involved in co-creating, testing, and evaluating an innovation in open, collaborative, multi-contextual, and real-world settings [5,6]. In contrast with traditional information systems research where organizational leverage exists to secure user participation, within the living lab approach the participation is usually voluntary, and the participation of the end-users needs to be encouraged [7]. However, the participants tend to drop-out of living lab activities before the project has ended [8, 9]. Participant drop-out might be due to an internal decision to stop the activity or to external environmental factors that cause them to terminate their engagement before completing the 
assigned tasks [10]. Such drop-out can occur in all phases of the ISD process, from contextualization, to testing, and to evaluation [11].

Despite the fact that keeping participants motivated is more challenging than motivating them to start participating in a project $[9,12]$, the literature still lacks an understanding on how to keep participants motivated and what actions are necessary to reduce the likelihood of user drop-out throughout the user engagement process $[1,8]$. Sustainable user engagement throughout the ISD process is deemed important due to factors such as time efficiency, cost efficiency, quality assurance, the value of an established mutual trust, and the participants' deep understanding about the project or activity $[9,13]$. In this study, we aim to determine which key factors are influential on participant drop-out behavior during the testing of an innovation in a living lab environment. We carried out an exploratory case study within a living lab setting to elicit as many influential factors on participant drop-out behavior as possible. In this case study, the participants were engaged in testing an innovation during the pilot phase the application's development. The paper summarizes the key lessons learned from our case study for how to reduce the likelihood of participant drop-out throughout the innovation process in a living lab setting, and it concludes with several avenues for future research in this field.

\section{Background}

User involvement in the ISD process had already had a long tradition when the participatory design was first introduced $[14,15]$. On the other hand, opening up the innovation process by involving different stakeholders such as individual users in different innovation activities is a key factor in ISD [16]. Open innovation research is strongly grounded in democratization and empowerment values, and it highlights the perspective of the users $[6,7]$. Therefore, users should be motivated to contribute to the projects [7]. However, finding motivated participants for long-term engagement in a project is not an easy task because they might tend to drop-out before completing the project or activity $[17,18]$. Habibipour et al. [11] carried out a comprehensive literature review and identified more than 30 influential factors on participant drop-out behavior that are associated with: (1) task design; (2) scheduling; (3) the participant selection process; (4) participant preparation; (5) implementation and testing processes; and (6) interactions with the participants. However, in the above-mentioned study, the authors did not focus on a specific phase or type of activity, and they extracted the drop-out reasons for all steps of the ISD process such as ideation, co-design or co-creation, and finally testing and evaluation. In this paper, we argue that this view is too general and that drop-out reasons need to be scrutinized at specific phases of the innovation process.

There have been attempts to present a user engagement process model that includes the variety of reasons for participant drop-out [18, 19]. For instance, Georges et al. [18] proposed the user engagement model for field trials, aiming to explain the factors that affect the engagement of end-users to test innovations in real-life environments. In this model, although they included some possible influential factors on user drop-out behavior in general terms such as perceived usefulness, perceived ease of use, 
uncertainty, and functional maturity, their analysis of these factors remained cursory, and this is something that current research needs to investigate in greater detail.

Although some studies have identified influential factors on participant drop-out behavior, there are contradictions among different research studies. For example, Kienle and Ritterskamp [20] recommend that the task should divided into subtasks with a fixed deadline per task, whereas Kobren et al. [21] claim that setting specific goals may be disadvantageous for user participation because participants immediately tend to drop-out upon finishing that goal. Therefore, the question is what the consequences are of a single deadline to complete all tasks compared to one deadline per task on participant drop-out behavior.

The main objective of this study, therefore, was to identify key influential factors on participant drop-out behavior during the testing of an innovation in a living lab environment, as well as the influence of fixed and flexible deadlines on participant drop-out behavior in the field test.

\section{Methodology}

In this study, we aimed to identify the factors influencing participant drop-out behavior throughout the process of testing an innovation in a living lab setting. An exploratory case study is the most suitable method for end-user studies because there is no contractual relationship between the subjects and the setting [22]. This approach enabled us to combine multiple sources of evidence as a means to ensure construct validity of the study. Triangulation of the data yielded stronger and more reliable conclusions compared with a single data source [22, 23].

Our case study research consisted of the four major steps as suggested by Yin [22]: designing the case study, preparing for data collection, collecting the evidence, and analyzing case study evidence.

\subsection{Study Design}

In this research, a user study was performed as part of an EU-FP 7 project called USEMP $^{1}$. The project is aimed at developing tools to enhance privacy management in online social networks. The DataBait tool is the result of the USEMP project, and this tool makes predictions of users' privacy dimensions by inferring the online social network profile from the user's data. The project adopted Facebook as the case. Moreover, the tool gives an indication of what can be inferred from the user's profile and the effects of his/her Facebook friends on their own privacy.

Participants were invited to participate in the development process of the DataBait application. This phase of the application development consisted of five sub-activities that we called MicroTasks (MicroTask1 to MicroTask5). Within each MicroTask, the participants tested each feature of the DataBait tool and they filled in a questionnaire after completing the assigned task. The MicroTasks focused on application usability,

\footnotetext{
${ }^{1}$ For an overview of the project and list of deliverables, please refer to: www.usemp-project.eu.
} 
and user's feedback acted as a formative evaluation approach to further improve the DataBait application.

\subsection{Preparation for Data Collection}

In the first step - the preparation phase - we developed a semi-structured online questionnaire (i.e., the drop-out questionnaire) to elicit open-ended responses from the participants who completely filled out the recruitment survey but did not complete all of the MicroTasks (i.e., "dropped out participants"). In the drop-out questionnaire, we were interested in knowing why those who signed up for the test dropped out before the activity or project ended. Thus, the questions were mainly focused on the participant's drop-out reason and other possible influential factors on their drop-out behavior such as their initial motivation to participate. The questionnaire was customized for two different groups of participants. The first group was the participants who filled out the recruitment survey but did not participate in the DataBait application test or dropped out after the first MicroTask. We categorized this group as "early dropped out participants" because the first MicroTask only involved general questions about the participants and their privacy preferences and were not related to the DataBait application. The second type of drop-out participants was the participants who had been involved in the DataBait test and who had completed two or more MicroTasks. We named this group "late dropped out participants" because they were truly involved in the DataBait application test before dropping out.

The majority of the test users were recruited through an invitation that was advertised twice on the university website. The second stage of user recruitment was to send an invitation to the users who had participated in the previous phase of the DataBait application testing and who had agreed to participate in later phases of the project. An advertisement was also posted on some of Swedish universities' public Facebook pages. A total of 118 participants showed interest and completely filled out the recruitment survey.

In order to investigate the influence of flexible timing on participant drop-out behavior, we classified participants into two main groups, both with 59 participants. Group1 were the participants who received all five MicroTasks together at one time with a single deadline. Group2 were the participants who received the tasks one at a time with a specific deadline per MicroTask. This categorization was done to enable us to investigate the influence of a single deadline compared to one deadline per task.

We also applied two different incentive structures [24]. In each of the above-mentioned groups, half of the participants were incentivized with an online voucher worth 300 SEK $(\simeq 30 €)$ after completing all five MicroTasks. Thus, if the participant did not complete all five MicroTasks, they were not paid anything. The other half of the participants were incentivized by periodic micro-incentives, which means that the participants who completed the first three MicroTasks were paid 100 SEK $(\simeq 10 €)$, those who completed the first four MicroTasks were paid 200 SEK $(\simeq 20$ $€)$, and those who completed all five MicroTasks were paid 300 SEK. 


\subsection{Collecting the Evidence}

The case study started in late June 2016 with a total duration of 34 days. In our study, qualitative data were gathered from three different sources: (1) direct observation of participants and their behavior during the test phase; (2) email communication with participants during the test phase regarding information about how to carry out the test, technical problems that occurred during the test, and other problems they experienced; and (3) an online semi-structured questionnaire carried out after the user study had ended. The drop-out questionnaire was sent to all participants who filled out the recruitment survey but did not complete the test. If the participant did not complete the assigned MicroTask within the scheduled time, reminders were sent out. If we did not hear from them within 3-4 days after that reminder, we considered them to be "dropped out participants". A reminder also was sent out for the drop-out questionnaire. Figure 1 show the execution timeline of this user study.

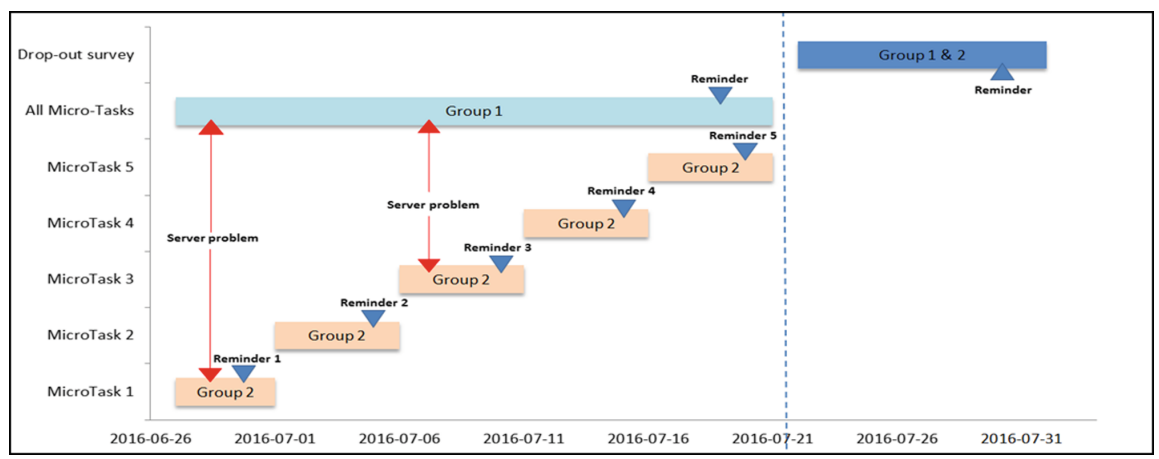

Fig. 1. Execution timeline of this user study.

\subsection{Analyzing Case Study Evidence}

The main analysis method employed in our study was qualitative data analysis of our direct observations, participant's feedback during their participation, and participants' responses to open-ended questions in the questionnaire. According to Yin [22], examining, categorizing, coding, and recombining evidence collected from multiple sources by different methods are the major steps of data analysis in a case study. To gain new insights about participant drop-out behavior, we started the data analysis in parallel with data collection by monitoring and documenting participants' behavior from the first day of the project. Thereafter, all participant feedback during the test was classified and coded by date and subject. We then combined these data with our observations of the project events such as reminders and server failures. Such observation were deemed important. For example, the server failures could potentially affect participants' motivation to remain or to drop-out of the user study. Finally, we classified and coded participants' answers to our questions related to their drop-out reasons and other influential factors on their participation behavior that were extracted from the qualitative questionnaire. In order to properly analyze the data and gain thorough 
insights, Microsoft Excel 2016 was used for coding and combining the collected information from the three waves of data collection.

\section{Results}

\subsection{Participation and Drop-Out Rate}

A total of 118 participants showed interest in participating in our user study and completely filled out the recruitment survey. Of these, 86 participants completed MicroTask1, 53 completed MircoTask2, 34 completed MicroTask3, 31 completed MicroTask4, and 27 completed MicroTask5 and reached the end of the user study. This resulted in 91 participants $(77 \%)$ who dropped out of our user study. Figure 2 shows the participation and drop-out rate.

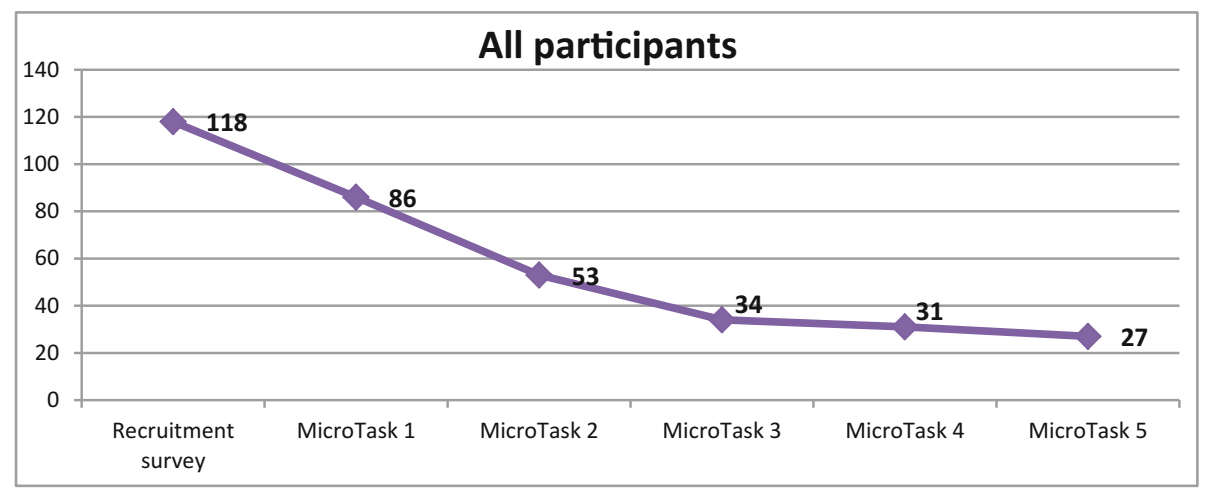

Fig. 2. Participation and drop-out rate

The drop-out rates were then compared between the two defined groups. The number of participants who reached the end of the test in Group1 was more than two times greater than in Group2. As it can be seen in Fig. 3, 19 participants in Group1 completed all five MicroTasks compared to only 8 participants in Group2 who fulfilled all the MicroTasks within the scheduled time.

Our results did not show any significant differences between participant behaviors with the two different method of receiving incentives.

\subsection{Drop-Out Questionnaire Results}

The drop-out questionnaire was sent to all 91 "dropped out participants" who filled out the recruitment survey but did not complete the test. In sum, we received 32 complete responses. Of these, 14 responses were from "late dropped out participants" and the other 18 responses were from the "early dropped out participants". 


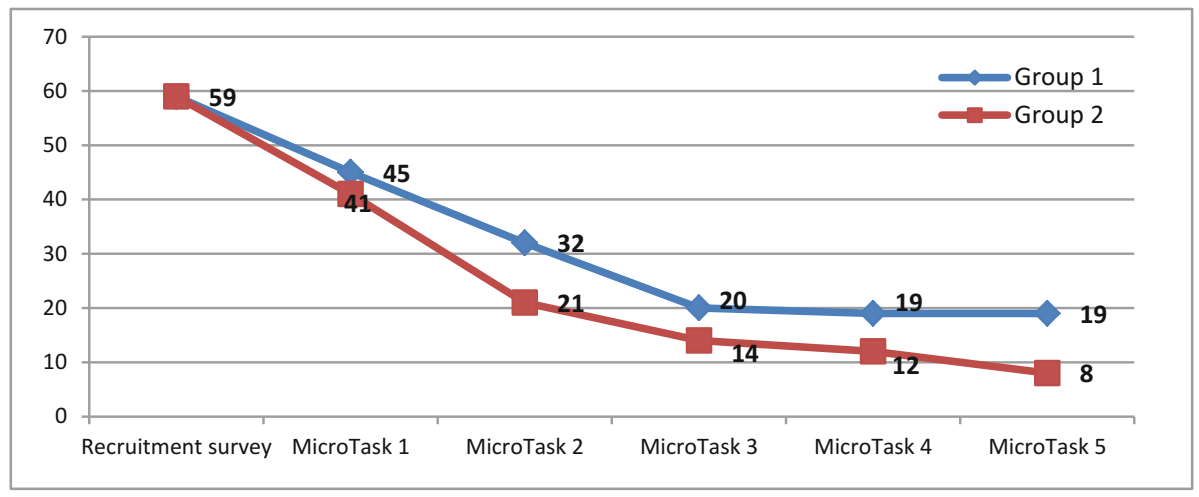

Fig. 3. Participant drop-out rate in the two groups

We first asked open questions about the initial motivation of participants to participate in the user study. The main motivation of half of the respondents (16 of 32) was curiosity about the research subject. Other motivations that were mentioned by the participants were financial reward, helping their university by contributing to research, learning something new, and having fun.

With regard to the "late dropped out participants", instability or non-functionality of the DataBait prototype was the most influential factor and was mentioned by six participants. They encountered many problems while trying to log in to the DataBait application. Some participants also complained that the MicroTasks were hard to understand, too long, exhausting, and the instructions difficult to follow. Inflexibility of the DataBait application due to incompatibility with the smartphone was the next affective factor on their drop-out decision. Limitation of access to the computer or Internet, insufficient number of reminders, too strict deadlines, summer holiday, and time limitations were other influential factors.

For "early dropped out participants", privacy concerns due to detailed personal questions and insecurity about the DataBait application were mentioned by seven participants. Similar to "late dropped out participants", the complexity of the MicroTasks besides the lack of clear instructions was also very influential in their motivation. The forgetfulness of the participants and their request to receive more than one reminder was another important factor. Summer holiday and the time intensity of the tasks were the next discouraging factors. Some participants also were dissatisfied with DataBait's incompatibility with the smartphone or non-functionality of the DataBait application when they tried to $\log$ in to the application. Table 1 shows the main drop-out reasons for both groups of dropped out participants.

\section{Data Analysis}

The results of our analysis show that there were many reasons for why participants dropped out of this user study. The analysis was conducted based on different sources of evidence, including direct observation, email communication, and the drop-out 
Table 1. Content analysis of responses to the open-ended question: 'What were your main reasons for dropping out of the DataBait user study?' (number of survey respondents: 32)

\begin{tabular}{l|l|l|l}
\hline Drop-out reason & $\begin{array}{l}\text { Late dropped out } \\
\text { participants }\end{array}$ & $\begin{array}{l}\text { Early dropped out } \\
\text { participants }\end{array}$ & Sum \\
\hline $\begin{array}{l}\text { Instability/non-functionality of the } \\
\text { prototype }\end{array}$ & 6 & 1 & 7 \\
\hline Too long tasks/exhausting & 3 & 3 & 6 \\
\hline $\begin{array}{l}\text { Prototype inflexibility/incompatibility } \\
\text { with smartphones }\end{array}$ & 3 & 1 & 4 \\
\hline Forgetfulness/insufficient reminders & 2 & 5 & 7 \\
\hline Hard to understand/too complicated & 2 & 3 & 5 \\
\hline Personal life problems & 2 & 1 & 3 \\
\hline $\begin{array}{l}\text { Limited access to the } \\
\text { computer/internet }\end{array}$ & 2 & 0 & 2 \\
\hline $\begin{array}{l}\text { Strict deadlines/deadlines too close to } \\
\text { each other }\end{array}$ & 2 & 0 & 2 \\
\hline Summer break/vacation & 1 & 4 & 5 \\
\hline Unclear instructions & 1 & 3 & 4 \\
\hline Time limitation & 1 & 2 & 5 \\
\hline Privacy concerns/personal questions & 0 & 5 & 2 \\
\hline Unsure about the app's security & 0 & 2 & 3 \\
\hline Other & 2 & 1 & \\
\hline
\end{tabular}

questionnaire. By coding the results of all three data sources, six categories seem to us to be the most meaningful way of organizing the factors influencing participant drop-out behavior within a living lab setting. These include the stability of the prototype, ease of use and understandability, privacy and security protection, flexibility or compatibility of the prototype, the effects of reminders, and timing issues. In the following, we discuss each of the following factors.

Stability of the Prototype. Most of the drop-outs were due to the instability of the prototype. We faced two major server failures during the test phase (see Fig. 1). This issue was highlighted mostly by the late dropped out participants, and they became exhausted because they were not able to $\log$ in to the system. In response to the question of "what were your main reasons for dropping out of the DataBait user study", we got responses such as: "Could not get the software to work, tried many times" and "The DataBait site did not work". The results of email communication as well as the drop-out questionnaire showed that some participants were also confused about the problem: "Do you have a server problems or do I have a bad memory? Did I do something wrong?" Moreover, if the prototype does not work as promised, it can lead to participant frustration. As one of the late dropped out participants stated: "It didn't work as planned".

Ease of Use and Understandability. Regarding the ease of use and understandability, we obtained answers like "Some things were hard to understand how they worked", "I did not understand what to do", "... exhausting! So many questions!", "Sometimes hard 
to understand. A bit complicated", "Tests were too difficult with too much to read", "Surveys were too difficult, boring", and "Tasks were too complicated" from both early and late dropped out participants. Some of the dropped out participants also argued that they were discouraged due to the lack of clear instructions on how to perform the MicroTasks. They expressed their discouragement by saying: "Did not find any help or guidelines when my problems occurred", "After completing the first questionnaire, [I] did not find information on how to proceed", "Clarify instructions ...", and "I signed up late, did not find sufficient information (and did not have time to ask for clarifications)".

Privacy and Security Protection. Some dropped out participants, especially early dropped out participants, expressed their concerns about their privacy by commenting: "... questions that were too personal", "... [I] didn't want to share my data...", "Insecure about how much data the application will be able to get", and "Not feeling certain about installing something I know little about".

Flexibility or Compatibility of the Prototype. In this category, we got responses from dropped out participants such as "I had problems reading in the DataBait interface. It was not compatible with my iPhone. I had to twist and turn the phone to be able to get a whole view", "Could not use my smartphone instead of my computer when on a trip...", and "I could not do the test on my iPhone. DataBait didn't work on the screen". Besides the compatibility issues, some participants also complained about accessibility problems. Because the test users should have installed an extension to their browser, they usually needed to have access to their own computer. Therefore, some participants were discouraged from remaining in the field test by saying: "I don't have access to a computer regularly" and "I was on a trip and I didn't bring my computer. Then I tried to use my smartphone to solve the final task but it did not work. I tried to use the hotel computer, but it did not work either...".

The Effect of Reminders. Another influential factor on participant drop-out rate in our field test was related to the participants' forgetfulness. For instance, an early dropped out participant in response to his drop-out reason stated: "Forgetfulness. I regularly forgot that I had signed up, and I simply did not remember to finish the test." We tried to overcome the problem of participant forgetfulness by sending one reminder for each MicroTask to the participants who did not fulfill the task within the scheduled time. However, some participants still argued that they needed more reminders to complete the tasks. For example, an early dropped out participant stated: “... write one more reminder mail - I know there actually was one, but sometimes there are just too many other mails and other things to do...". This issue was also mentioned by some of the late dropped out participants, as one said: "Give more reminders. I understand that there are people that maybe don't want constant reminders, but I am one of those who really need to be reminded - I have a terrible memory and I keep prioritizing things that I probably shouldn't. Maybe give participants options for how often they should be reminded? (I could seriously use daily reminders, at least when the deadline is closing in)".

Timing Issues. Regarding the timing issues, the reasons for participants to drop-out of the field test were related to the inflexibility of the scheduling as well as an inappropriate time of the year. Some of the dropped out participants in Group2 mentioned that 
the tasks were too close together by stating: "The surveys had numerous tasks that needed a lot of time [...]. Also give more time between surveys". The importance of flexibility in timing became more apparent to us when we received some comments from those who asked for longer time to complete the assigned tasks. We got responses such as: "The timing was my biggest problem, if I had a few days extra it would have not been any problem", "At the moment I don't have the time. But next week I do so if you could wait until then I will gladly be a participant", and "The surveys were too close together and that in addition to a very stressful period at work, I didn't get the time to do it".

Concerning the time of the year, we got some responses such as "I received one of the assignments while I was on vacation and couldn't access my computer. By the time I got home I was behind and couldn't continue", “... Vacation. I went on vacation in the summer, which limited my access to computers and the Internet", and "Bad idea having a test during the vacation months".

\section{Discussion and Conclusion}

Our study contributes to previous research by featuring key negative factors that influence the motivation of participants to stay engaged and that lead to participant drop-out when testing an innovation in a living lab setting.

A notable finding of our case study was related to the stability of the prototype, especially when the participants had access to all of the subtasks. For example, participants in Group1 started to test the application with the intention to complete all five MicroTasks as soon as possible. The rush to use the application caused the server to fail to respond to any request on two occasions both for two days. Therefore, it is of crucial importance to verify the stability of the prototype to prevent server overload behavior, especially when the number of test users is relatively high, otherwise it can lead to participant frustration. One way to overcome this type of problem is to make the participants aware and well informed that the prototype is not as stable and reliable as a commercial technology, which is in line with Taylor et al.'s [25] recommendation.

Our finding also supports Zheng et al.'s [26] finding that analyzability (i.e., the degree of task complexity as well as the availability of information about the tasks) is positively associated with the users' motivation. If the tasks are not simple enough, some participants are not able to understand the task [21] and consequently will not enthusiastically engage in the process. To avoid complexity, a clear and accessible guideline would minimize the risk of confusion and resulting discouragement. Although guidelines and instructions on how to perform the MicroTasks had been prepared for the participants, some of them were not able to find and use them. Therefore, the organizers of the field test need to make the participants aware of the whole engagement process and to create guidelines and instructions that in addition to being comprehensible are easily accessible and available.

When it comes to privacy and security concerns, our findings were consistent with previous studies that privacy protection is positively associated with sustainable user engagement [27, 28]. As Georgeos et al. [29] argue, users are concerned about the security of their information and they might drop-out of the project if they have to fill in 
their personal information in a system or an application, especially when the system is under development and thus is not highly stable. Another interesting observation about the privacy concerns was that a total of 205 participants started to fill out the recruitment survey, but only 118 of them completed this survey. Most of them stopped completing the survey when they were asked to give a link to their Facebook account in the recruitment survey. One plausible explanation is that they were concerned about their identity and preferred to be an anonymous contributor.

Regarding the flexibility of the timing, the number of participants who reached the end of the test in Group1 was almost two times greater than in Group2. This finding aligns with Wilson et al.'s [30] finding that users prefer to carry out the tasks at their own pace, especially when they are participating in a multi-task user study. Interestingly, despite the fact that the total time for carrying out all five MicroTasks was equal in both Group1 and Group2, we did not receive any comment from the dropped out participants in Group1 regarding the time limitation because they were able to complete the tasks at their own pace. However, as mentioned earlier, giving all tasks together might cause other problems in the field tests such as overload on the server, as we experienced in our case.

In consideration of the time of the year, we faced many drop-outs due to summer holiday and vacation time because the field test was conducted in the summer. Therefore, the organizers of a user study should consider that the test users might not have access to their computer or to the Internet during their vacation period. Moreover, in order to reduce the likelihood of participants' forgetfulness, a sufficient number of reminders must be set in the schedule of the field tests. As our direct observations showed, the participation rate immediately increased after sending a reminder to the test users, and thus the effects of sending reminders need to be further investigated.

Regarding different kinds of incentivization, although many of dropped out participants mentioned monetary reward as their main motivation to participate in the test, our results were contradictory to [24], and there were no significant differences in participant drop-out behavior with two different method of receiving incentives. One possible explanation for this is the duration of the user study, which was relatively short and thus the periodic micro-incentives might not have had an effect. Moreover, the amount of the financial reward in this case was very small and thus might not have made a difference in the drop-out rate.

One limitation of this study is that the factors extracted in our study might be case or project specific, and they need to be tested in other projects. For example, the issue of privacy was very crucial in our project because the developed application needed to get access to the user's Facebook data. Another limitation was that cultural factors were likely to be influential. Our sample participants included only Swedish participants, and employing a mixed panel might have led to different results. The relatively low number of responses to our drop-out questionnaire (32 of 91 dropped out participants) is also a limitation of this study.

This study also opens opportunities for future research. As O'Brien and Toms [10] have introduced re-engagement as one of the core concepts of their user engagement process model, an interesting topic for further research would be to clarify how and why user motivation for engaging and staying engaged differ. More specifically, it 
would be interesting to identify how the organizers of a user study can re-motivate the dropped out participants to re-engage in the study.

Acknowledgments. This work was funded by the European Commission in the context of the FP7 project USEMP (Grant Agreement No. 611596), the Horizon 2020 project PrivacyFlag (Grant Agreement No. 653426), and the Horizon 2020 project U4IoT (Grant Agreement No. 732078). We would also like to thank all participants who helped us with their feedback during the application test and the post-test survey.

\section{References}

1. Leonardi, C., Doppio, N., Lepri, B., Zancanaro, M., Caraviello, M., Pianesi, F.: Exploring long-term participation within a living lab: satisfaction, motivations and expectations. In: Proceedings of the 8th Nordic Conference on Human-Computer Interaction: Fun, Fast, Foundational, pp. 927-930. ACM, New York (2014)

2. Bano, M., Zowghi, D.: A systematic review on the relationship between user involvement and system success. Inf. Softw. Technol. 58, 148-169 (2015)

3. Lin, W.T., Shao, B.B.: The relationship between user participation and system success: a simultaneous contingency approach. Inf. Manag. 37, 283-295 (2000)

4. Chesbrough, H.: Open innovation: a new paradigm for understanding industrial innovation. In: Chesbrough, H., Vanhaverbeke, W., West, J. (eds.) Open Innovation: Researching a New Paradigm, pp. 1-12. Oxford University Press, Oxford (2006)

5. Ståhlbröst, A.: Forming future IT: the living lab way of user involvement (2008). http:// epubl.ltu.se/1402-1544/2008/62/index-en.html

6. Bergvall-Kareborn, B., Holst, M., Stahlbrost, A.: Concept design with a living lab approach. In: 42nd Hawaii International Conference on System Sciences, 2009. HICSS 2009, pp. 1-10. IEEE (2009)

7. Ståhlbröst, A., Bergvall-Kåreborn, B.: Voluntary contributors in open innovation processes. In: Eriksson-Lundström, J.S.Z., Wiberg, M., Hrastinski, S., Edenius, M., Ågerfalk, P. J. (eds.) Managing Open Innovation Technologies, pp. 133-149. Springer, Berlin (2013)

8. Ogonowski, C., Ley, B., Hess, J., Wan, L., Wulf, V.: Designing for the living room: long-term user involvement in a living lab. In: Proceedings of the SIGCHI Conference on Human Factors in Computing Systems, pp. 1539-1548. ACM, New York (2013)

9. Ley, B., Ogonowski, C., Mu, M., Hess, J., Race, N., Randall, D., Rouncefield, M., Wulf, V.: At home with users: a comparative view of living labs. Interact. Comput. 27, 21-35 (2015)

10. O'Brien, H.L., Toms, E.G.: What is user engagement? A conceptual framework for defining user engagement with technology. J. Am. Soc. Inf. Sci. Technol. 59, 938-955 (2008)

11. Habibipour, A., Bergvall-Kåreborn, B., Ståhlbröst, A.: How to sustain user engagement over time: a research agenda. In: Proceedings of Twenty-Second Americas Conference on Information Systems (AMCIS). AIS, San Diego (2016)

12. Pedersen, J., Kocsis, D., Tripathi, A., Tarrell, A., Weerakoon, A., Tahmasbi, N., Xiong, J., Deng, W., Oh, O., De Vreede, G.-J.: Conceptual foundations of crowdsourcing: a review of IS research. In: 46th Hawaii International Conference on System Sciences (HICSS), pp. 579-588. IEEE (2013)

13. Sambamurthy, V., Kirsch, L.J.: An integrative framework of the information systems development process. Decis. Sci. 31, 391-411 (2000) 
14. Bansler, J.: Systems development research in Scandinavia: three theoretical schools. Scand. J. Inf. Syst. 1, 3-20 (1989)

15. Iivari, J., Lyytinen, K.: Research on information systems development in Scandinaviaunity in plurality. Scand. J. Inf. Syst. 10, 135-185 (1998)

16. Chesbrough, H., Crowther, A.K.: Beyond high tech: early adopters of open innovation in other industries. R\&D Manag. 36, 229-236 (2006)

17. Kaasinen, E., Koskela-Huotari, K., Ikonen, V., Niemelä, M., Näkki, P.: Three approaches to co-creating services with users. In: Spohrer, J.C., Freund, L.E. (eds.) Advances in the Human Side of Service Engineering, pp. 286-295. CRC Press, Boca Raton (2013)

18. Georges, A., Schuurman, D., Baccarne, B., Coorevits, L.: User engagement in living lab field trials. Info 17, 26-39 (2015)

19. Habibipour, A., Bergvall-Kåreborn, B.: Towards a user engagement process model in open innovation. In: ISPIM Innovation Symposium. The International Society for Professional Innovation Management: Moving the Innovation Horizon. ISPIM (2016)

20. Kienle, A., Ritterskamp, C.: Facilitating asynchronous discussions in learning communities: the impact of moderation strategies. Behav. Inf. Technol. 26, 73-80 (2007)

21. Kobren, A., Tan, C.H., Ipeirotis, P., Gabrilovich, E.: Getting more for less: optimized crowdsourcing with dynamic tasks and goals. In: Proceedings of the 24th International Conference on World Wide Web, pp. 592-602. International World Wide Web Conferences Steering Committee, Republic and Canton of Geneva, Switzerland (2015)

22. Yin, R.K.: Case Study Research: Design and Methods. SAGE, Los Angeles (2008)

23. Benbasat, I., Goldstein, D.K., Mead, M.: The case research strategy in studies of information systems. MIS Q. 11, 369-386 (1987)

24. Musthag, M., Raij, A., Ganesan, D., Kumar, S., Shiffman, S.: Exploring micro-incentive strategies for participant compensation in high-burden studies. In: Proceedings of the 13th International Conference on Ubiquitous Computing, pp. 435-444. ACM, New York (2011)

25. Taylor, N., Cheverst, K., Wright, P., Olivier, P.: Leaving the wild: lessons from community technology handovers. In: CHI 2013 Proceedings of the SIGCHI Conference on Human Factors in Computing Systems, pp. 1549-1558. ACM, New York (2013)

26. Zheng, H., Li, D., Hou, W.: Task design, motivation, and participation in crowdsourcing contests. Int. J. Electron. Commer. 15, 57-88 (2011)

27. Ståhlbröst, A., Padyab, A., Sällström, A., Hollosi, D.: Design of smart city systems from a privacy perspective. IADIS Int. J. WWW Internet 13, 1-16 (2015)

28. Padyab, A.M.: Getting more explicit on genres of disclosure: towards better understanding of privacy in digital age (research in progress). In: Nor. Konf. Organ. Bruk Av IT. 22 (2014)

29. Georges, A., Schuurman, D., Baccarne, B.: An exploratory model of the willingness of end-users to participate in field tests: a living lab case-study analysis. In: Proceedings of Open Living Lab Days 2014, Amsterdam, The Netherlands (2014)

30. Wilson, S., Bekker, M., Johnson, P., Johnson, H.: Helping and hindering user involvement - a tale of everyday design. In: CHI 1997 Proceedings of the ACM SIGCHI Conference on Human factors in computing systems, pp. 178-185. ACM, New York (1997) 